\title{
CAMBIO SOCIAL Y TOTALIDAD
}

\section{SOCIAL CHANGE AND TOTALITY}

Mg. Esteban Torres (esteban.tc@gmail.com) Centro de Estudios Avanzados, Universidad Nacional de Córdoba (Córdoba, Argentina)

\begin{abstract}
The general purpose that guides this article is to contribute to the critique and study of the possibilities of reformulation of the category of social totality and social change or some of their central elements, as theoretical alternatives to think again about the urgent and historical matter of development (mainly in its economic dimension), in order to face the new cultural and political challenges present in Latin American societies.
\end{abstract}

Keywords: totality, change, social, theory, development.

\section{Resumen}

Orienta el desarrollo del artículo el propósito de contribuir a la crítica y al estudio de las posibilidades de reformulación de la categoría de totalidad social y cambio social o bien de elementos centrales de las mismas, como alternativas teóricas para repensar la urgente e histórica cuestión del desarrollo (principalmente en su dimensión económica) y con ello hacer frente a los nuevos desafíos culturales y políticos que se presentan en las sociedades latinoamericanas.

Palabras clave: totalidad, cambio, social, teoría, desarrollo.

\section{Introducción}

En el presente artículo (1) proponemos un primer registro exploratorio y acotado de ciertos problemas metodológicos que atañen a las teorías del cambio social. Nuestra atención se concentra en una articulación específica: el vínculo entre el cambio social y la categoría de totalidad (2). El avance en el desarrollo de dicho aspecto resulta fundamental para las pretensiones de validez de la investigación social centrada en la categoría de cambio social (3), relativamente desvalorizada en las ciencias sociales desde mediados de la década del 80 del siglo pasado.

Mientras que el accionar de la crítica científica, en los términos clásicos de Kuhn, suele precipitar la revolución paradigmática, logrando imponer una propuesta teórica superadora de la anterior (Kuhn 2005), suele igualmente suceder que muchos de los problemas planteados en el pasado fueron suspendidos pero no superados teóricamente, o al menos no fueron agotados en los términos de sus propias pretensiones de verdad. En este caso, no es la falta de respuestas desde el paradigma vigente la que provoca la crisis, como señala Kuhn, sino que se impone una sustitución por "accidente", por lo general a partir de la instauración de nuevos proyectos políticos, que interrumpen y/o destruyen los procesos científico-técnicos existentes, vigentes y exitosos, eventualmente por considerarlos una amenaza para el nuevo proyecto. Se trata de una lógica que trasciende y se impone a la lógica institucional de las ciencias en sentido estricto. Entendemos que el programa de estudios del cambio social se inscribe plenamente en dicha realidad. Si bien registramos 
Torres, E. 2011. Cambio Social y Totalidad

producciones desde la década del noventa en adelante (Mc Michael 2004, Roberts y Hite 2000, Francisco 1997, Appelbaum y Chambliss 1997, Sztompka 1995, Morrow 1995, Smelser y Haferkamp 1992, Y So 1990, Harrison 1988, etc.) los trabajos más relevantes se desarrollan en su mayoría en el período 1950-1970, con algunas contribuciones aisladas en la primera mitad de la década de los 80 (Giddens 1984, Boudon 1985, etc.).

El período que transcurre entre 1945 y 1968 corresponde no sólo a la edad de oro del crecimiento económico del capitalismo, sino también de las fuerzas de liberación nacional y de las fuerzas antisistémicas, que en sus diferentes variantes alcanzaban el poder estatal a lo largo y ancho del mundo (Wallerstein 1994). A partir de allí, como se reconoce en la historiografía social y política mundial, a principio de los años setenta -más concretamente de 1968- se inicia un proceso de cambio a nivel mundial que sienta las bases para el desarrollo del proyecto social, económico y cultural del neoliberalismo, y que siguiendo la reflexión previa, podríamos concebir como "accidente" para buena parte de las investigaciones sociales (y los investigadores) centradas en la pregunta por el cambio social. Ese es el motivo principal por el cual el lector encontrará en el presente artículo bibliografía en su gran mayoría de dicha época.

El comienzo del fin de la hegemonía discursiva de la posmodernidad o en términos de Grunner (2002), de las pequeñas historias, abre nuevamente las ciencias sociales a las teorías de la modernidad y con ellas a las teorías del cambio social. Ahora bien, ellas podrán aspirar a recobrar valor y pertinencia sólo en la medida en que logren asumir sus fracasos más resonantes, integrando las contribuciones de la crítica, entre ellas los aciertos de la crítica posmoderna.

La restauración del valor analítico y práctico de la investigación sociológica sobre la modernidad, el cambio social y el desarrollo implica una esmerada revisión de muchas de sus premisas fundamentales. Para ello, no alcanzaría con el reconocimiento de la elusividad que plantea el concepto (London 1996) o su carácter abstracto (Tilly, en Stompka 1995:45). Si bien la categoría de cambio social se inscribe en buena medida al interior del vínculo entre la teoría social y las distintas perspectivas evolucionistas (entre ellas el materialismo histórico), no acordamos con Nisbet cuando afirma de modo taxativo que la noción de cambio social designa un proyecto que se propone la búsqueda de regularidades del cambio y la determinación de leyes de evolución del sistema social (Nisbet, en Boudon 1985:322). Veremos que la noción de cambio social no tiene por qué reducirse a las premisas sociológicas ortodoxas de la búsqueda de regularidades y el establecimiento de "leyes". Adherimos a la visión de Boudon cuando afirma que la filiación de las teorías del cambio social con la filosofía de la historia y la sociología histórica es tan sólo eventual y probable (Boudon 1985). Una posición similar sostiene Giddens (1984) al expresar que no es necesario suscribir a dicha fórmula para abordar la cuestión del cambio social, y luego Tilly cuando señala que no hay un único proceso maestro de cambio social sino procesos fragmentarios a distintos niveles de complejidad, a veces paralelos, otros contrapuestos o solapados (Tilly, en Stompka 1995:46).

Por otra parte, todo intento de actualización de las teorías del cambio social debe tener presente los límites epistemológicos que actualmente exigen legitimar la coexistencia de diferentes discursos teóricos. Sin necesidad de adoptar la actitud relativista de Boudon, es recomendable acordar con éste respecto a la necesidad de ejercer la crítica y preguntarse sobre las condiciones de posibilidad del programa de estudios resumido en el concepto de cambio social (Boudon 1985:323), inscripto en gran medida en la tradición del funcionalismo-estructuralista, así en el marxismo. En resumidas cuentas, se trata de intentar recuperar las perspectivas generales del cambio social desde una perspectiva abierta y reflexiva, atenta a los avances 
epistemológicos del "postmodernismo". Si bien no es una empresa sencilla, consideramos que las visiones totalizantes, complejas y multidimensionales de lo social, propias de la teoría social moderna, pueden ser actualizadas y "domesticadas" -diría Althusser-con éxito.

\section{El cambio social: Aspectos generales}

En principio no existen acuerdos generales sobre los aspectos de conocimiento y de método que implica la aproximación al estudio del cambio social. Tampoco se evidencian registros comunes sobre las dificultades principales que dicha empresa acarrea, pese a las voluminosas críticas vertidas sobre el tema a partir de mediados de la década de 1980 del siglo pasado. Como luego veremos, algunos de los problemas surgen en relación con el alcance de la teoría. Entre otras cuestiones, como ya señalaban Pettigrew y Back a mediados de los años 1960, "es difícil a menudo determinar si algún cambio particular constituye una encrucijada en la cual la modificación con ese sentido se mantendrá como un ciclo de larga duración, una fluctuación breve, parte de una tendencia evolutiva, o alguna otra manifestación del cambio radical" (Pettigrew y Back 1967:68). Otra de las dificultades atañe a la propia de definición del cambio social, con la especificidad que le añade la generalización de los diversos usos heurísticos que se hace de la categoría, enlazados frecuentemente con disputas estrictamente ideológicas (ver en Puigbo 1966:1413). Los estudios del cambio social también experimentan problemas para discernir lo que cambia y lo que no, la determinación de los factores intervinientes en el proceso, la identificación del conjunto de los actores sociales involucrados en el proceso de cambio, y un largo etcétera de aspectos vitales para la comprensión y transformación de los procesos sociales.

Sólo a modo ilustrativo, a partir del trabajo de recopilación de varios autores, podemos identificar una serie de preguntas que comparten gran parte en las investigaciones del cambio social. ¿Qué es lo que está cambiando? (Moore 1966:56, Wright Mills 1964:96) ¿Cómo cambia? (Wright Mills 1964:96) ¿En qué dirección? (Wright Mills 1964:96) ¿Con qué velocidad? (Wright Mills 1964:96) ¿Por qué? (Wright Mills 1964:96) ¿Cuál es la naturaleza o la característica del cambio? (Nisbet 1979:10, Eisenstadt 1970:10) ¿En qué consiste su proceso (Nisbet 1979:10) ¿Qué forma adquiere? (Nisbet 1979:10) ¿Cuáles son sus consecuencias? (Nisbet 1979:10) ¿Qué hace a los cambios socialmente importantes? (Jaguaribe 1972:71) ¿Cuáles son las condiciones que contribuyen a la importancia social del cambio? (Jaguaribe 1972:71) ¿Qué actor o sector es el que aparece primero? (Etzioni 1964:429) ¿Cómo se interrelacionan los diferentes procesos de cambio? (Eisenstadt 1970:10, Etzioni 1964:429). Dichas preguntas, aparentemente simples, implican muchas teorías contradictorias. No existen respuestas generales satisfactorias, pero sí formas generales de formular respuestas.

Como luego veremos, la recuperación de la pregunta por el cambio social desde la teoría social remite en gran medida a la necesidad de revisión de la perspectiva marxiana y los diferentes enfoques marxistas y neomarxistas, pero igualmente no puede obviar las referencias al dominio intelectual en occidente de las corrientes funcionalistas y estructural-funcionalistas durante la segunda mitad del siglo 20, cuyos abordajes de la cuestión del cambio social -centradas en la idea de orden e integración social- estuvieron en diálogo crítico con las primeras.

\section{Cambio social y totalidad}

Sabemos que el concepto de totalidad o bien de totalidad social es una categoría central de los principales métodos de conocimiento del cambio social. Entre ellos, las visiones dialécticas de la totalidad y la 
Torres, E. 2011. Cambio Social y Totalidad

totalización (4), principalmente de inspiración hegeliana y marxiana, son las que sustentan el espectro más amplio de perspectivas filosóficas y sociales que buscan conocer el conjunto de lo social a partir de sus procesos. Dichas perspectivas fueron fuertemente combatidas desde diferentes doctrinas, pero en especial desde el estructural-funcionalismo. Ahora bien, la investigación del cambio social no necesariamente debe someterse a la integración de dicho método, así como tampoco la noción de totalidad es propiedad exclusiva de dichas dialécticas. No es objetivo de este punto presentar un análisis exhaustivo de las diferentes categorías clásicas y contemporáneas de la totalidad o totalidad social, ni tampoco dar cuenta de las diferentes construcciones metodológicas más o menos sistemáticas y creativas que integraron o integran dicho perspectiva en los estudios sociales, más específicamente en las investigaciones sobre el cambio social. Nos ocuparemos, en cambio, de dos aspectos que consideramos claves: a) los argumentos principales a partir de los cuáles se reconoce la utilidad de la categoría; y b) una discordancia específica que domina los principales debates en torno al concepto de totalidad, a su validez como método, y a las condiciones para la apropiación efectiva de la categoría en tareas de investigación social: la cuestión de si el concepto de totalidad hace referencia a todos los hechos o bien a una perspectiva condicionada de todos los hechos capaces y posibles de ser registrados por un actor o conjunto de actores en una situación concreta. Nos concentraremos principalmente en este último punto, a partir del cual pretendemos poder establecer un registro inicial de las alternativas de significado que se podrían poner en juego al momento de fijar el método de análisis del cambio social.

El primero de los aspectos roza el sentido común del científico social, pero no por ello podemos excluir su tratamiento. Muchas de las visiones proclives a reconocer las ventajas analíticas del concepto han logrado establecer un diálogo constructivo con el marxismo. Ahora bien: ¿cuáles serían estas ventajas? En líneas generales se sostiene que la aproximación a cierta explicación del cambio social desde una noción de totalidad posibilita la superación de las visiones parceladas y fragmentadas del proceso social, propias de la compartimentación positivista de las ciencias sociales, que debilita la comprensión de la realidad y su potencia de actuación social sobre ella. Como señala Wright Mills, "el acento en problemas prácticos, fragmentarios, tiende a atomizar los objetivos sociales. Los estudios así informados no se integran en conjuntos suficientemente amplios como para servir a la acción colectiva, con la capacidad y la intención de realizar esa acción" (Wright Mills en Puigbo 1966:21). En la misma dirección se expide Morin, al considerar que los "pensamientos parciales y mutilantes conducen a acciones parciales y mutilantes; un pensamiento unidimensional desemboca en un hombre unidimensional" (Morin en Ciurana 1999:123).

Cuando se conoce parcialmente, sin ver lo que "está tejido en conjunto" se puede olvidar o ignorar las consecuencias del propio conocimiento, disminuyendo la posibilidad de interactuar como sujeto social. En este sentido, siguiendo a Mac Iver, "el cambio tiene sus repercusiones sobre la situación total, fundiéndose con las fuerzas del cambio dentro de ella" (Mac Iver 1949:219). La investigación de las causas del cambio social en la perspectiva de Mac Iver nunca puede llevarse a cabo a menos que abarque toda la pauta de relaciones dentro de la cual surge el fenómeno del cambio (Mac Iver 1949:188). Junto a la posibilidad de superar las percepciones fragmentadas de los procesos sociales, una visión de totalidad (en los términos dialécticos de Adorno) permitiría incluso razonar esa división como producto de una forma histórica específica de regulación social de los objetos, hechos y relaciones sociales (5).

En cuanto al segundo punto, el más importante, partimos de constatar que cualquier método de investigación del cambio social que se estructure a partir de la relación con cierta noción de totalidad o totalidad social se enfrenta con un conjunto de problemas de difícil solución. Como ya mencionamos, quizás 
el aspecto más crítico se centra en la cuestión de si el concepto de totalidad hace referencia a todos los hechos o bien a una perspectiva condicionada de todos los hechos capaces y posibles de ser registrados por un actor o conjunto de actores en una situación concreta, incorporando un sentido de totalidad abierta. Como veremos a continuación, en torno a este tema se constata uno de los principales desencuentros epistemológicos entre el marxismo (en sus diferentes expresiones) y el estructural-funcionalismo: mientras que los primeros propusieron nuevas versiones de la categoría, en vistas a su conservación, los segundos actuaron a favor de su exclusión, a partir del rechazo de su versión dogmática original. Aquí sólo revisaremos algunas de las posiciones más importantes.

Comencemos con las citas de ciertas perspectivas marxistas, comprometidas con la totalidad social. En el plano filosófico, siguiendo la perspectiva de Kosik, la totalidad es la realidad como un todo estructurado y dialéctico, y ésta no significa todos los hechos: "Pero, en verdad, la totalidad no significa todos los hechos. Totalidad significa: realidad como un todo estructurado y dialéctico, en el cual puede ser comprendido racionalmente cualquier hecho (clases de hechos, conjunto de hechos) [...] Los hechos son conocimiento de la realidad si son comprendidos como hechos de un todo dialéctico, esto es, si no son átomos inmutables, indivisibles e inderivables, cuya conjunción constituye la realidad, sino que son concebidos como partes estructurales del todo. Lo concreto, o sea, la totalidad, no es, por tanto, todos los hechos, el conjunto de ellos, el agrupamiento de todos los aspectos, cosas y relaciones, ya que en este agrupamiento falta aún lo esencial: la totalidad y la concreción (Kosik 1963:55).

A partir del empleo de una argumentación similar, hay quienes optan, como Badiou, por definir a la totalidad como un conjunto abierto, como totalidad abierta, en oposición a la totalidad cerrada: "El fondo de la cuestión estriba en saber si por totalidad entendemos un conjunto cerrado o un conjunto abierto. Es la cuestión filosófica introducida desde hace tiempo, particularmente por Bergson: la oposición entre comunidad cerrada o abierta. La crítica de la totalidad viene a decir que cualquier totalidad humana, viva, positiva y, por tanto, con un auténtico valor, no puede ser nunca una totalidad cerrada, sino siempre una totalidad abierta, a los otros, a la historia, al mundo. Por tanto, tampoco se puede decir que haya valores para todos los elementos de la totalidad porque 'todos los elementos de la totalidad' no quiere decir nada, la totalidad no es cerrada. Si la totalidad está abierta, nunca se tienen todos los elementos de la totalidad. Para ello haría falta que esa totalidad fuese cerrada" (Badiou 2006:21).

A las referencias mencionadas sumamos la identificación que establece Sartre entre totalidad y totalización. Éste tiende igualmente a enfatizar el carácter procesual e incompleto de la totalidad, y con ello ofrece pistas que permiten circunscribir -aunque no simplificar- el marco de posibles usos del concepto para la interpretación del cambio social. En aras de la claridad, pedimos permiso al lector para volver a citar en extenso: "Así la totalización tiene el mismo estatuto que la totalidad: a través de las multiplicidades prosigue este trabajo sintético que hace de cada parte una manifestación del conjunto y que lleva al conjunto a si mismo por la mediación de las partes. Pero es un acto en curso y que no se puede detener sin que la multiplicidad vuelva a su estatuto original. Este acto dibuja un campo práctico que, como correlativo indiferenciado de la praxis, es la unidad formal de los conjuntos que se tienen que integrar; en el interior de ese campo práctico, trata de operar la síntesis más rigurosa de la multiplicidad más diferenciada: así, por un doble movimiento, la multiplicidad se multiplica hasta el infinito, cada parte se opone a todas las demás y al todo en vías de formación, mientras la actividad totalizadora aprieta los lazos y hace de cada elemento diferenciado su expresión inmediata y su medición en relación con los otros elementos" (Sartre 1960:192). 
De esta forma, la totalidad existente de Sartre -en consonancia con el sentido abierto y procesual esbozado- se expresa como totalidad destotalizada: "la realidad del objeto colectivo (...) manifiesta que la totalización no está terminada nunca y que la totalidad de existir lo es a título de 'totalidad destotalizada'” (Sartre 1960:19). La insistencia respecto al dinamismo y la historicidad de esa totalización recuerda que se trata siempre de un proceso abierto, nunca logrado plenamente.

Con ciertos reparos, podríamos igualmente citar en este primer grupo a Walter Benjamin. Para ello deberíamos reconocer una diferencia importante, ya que éste no se ocupa de formular, no al menos de modo central, un concepto alternativo de totalidad. Si bien es a partir de postular la desaparición de la "totalidad orgánica" que el autor se concentra en la observación y el análisis de los fragmentos (de lo pequeño, los detalles, sobre lo cual se asienta su diferencia metodológica respecto a la dialéctica de Hegel y Marx), contra la interpretación culturalista de Sarlo (2009) es necesario destacar que su atención en lo minúsculo se orienta al descubrimiento del "acontecimiento histórico total". Su objetivo fundamental pasa entonces por aplicar a la historia el principio del montaje y de este modo "descubrir en el análisis del pequeño momento singular el cristal del evento total” (Benjamin 2004:575). Benjamin se aferró, en los Pasajes, a la idea de una fisiognómica materialista, aunque la concibiera como complemento o ampliación de la teoría marxista. La fisiognómica deduce del exterior el interior, descifra la totalidad del detalle, representa en lo particular lo general (Benjamin 2004). Por lo tanto, el proyecto de Benjamin reconoce el valor de la totalidad social, entendido ésta como una totalidad no orgánica, a partir de la atención en sus fragmentos, sus hechos minúsculos, en los productos de la sociedad.

El último caso que mencionaremos es el de Emmanuel Levinas, el único autor no marxista de la selección. Al igual que Benjamin, éste opta por no recodificar la noción ortodoxa de totalidad, sino más bien desarrollar una nueva noción, la de infinito, como contraposición a la primera, y que guarda fuerte similitudes con los conceptos renovados de totalidad ya mencionados. Dicha noción de Infinito reacciona a la Totalidad a partir del reconocimiento de la alteridad, fundamentada en términos éticos. Lo infinito, para Levinas, rompe con la idea de totalidad que encierra al Mismo y lo Otro, pero lo hace como un "más allá» que se expresa en el interior de la historia y de la experiencia. Por otra parte, Levinas considera que sólo a partir de la totalidad podemos acceder a una situación en la que ésta se fractura: "se puede acceder a partir de la experiencia de la totalidad a una situación en la que la totalidad se quiebra en tanto que esta situación condiciona la totalidad misma. Tal situación es el resplandor de la exterioridad o la trascendencia en el rostro del Otro (Autrui)" (Levinas 2002:107).

La relación de lo Mismo y del Otro, que no llega a totalizarse en una síntesis posterior, se puede configurar como tal gracias a que se mantiene la heterogeneidad absoluta de los términos. El Otro no puede ser pensado como lo Mismo puesto enfrente sin que asistamos a la destrucción radical de su alteridad, cayendo irremisiblemente en la lógica de la Totalidad. En este punto, según Dussel, se produce una cierta equivocidad en el pensamiento levinasiano que surge de la consideración del otro como lo absolutamente Otro (Dussel 1974). Podemos decir entonces que a partir del reconocimiento de la alteridad, el esfuerzo de Levinas se orienta a la construcción de aquellas mediaciones que permitirían establecer una nueva totalidad al servicio del Otro.

Al conjunto de posiciones mencionadas hasta aquí, en su amplia mayoría de inspiración marxista, se oponen algunos autores que podríamos inscribir en las corrientes estructural-funcionalistas, identificadas en muchos casos con las teorías de la modernización. El argumento metodológico más extendido hace hincapié en la 
Torres, E. 2011. Cambio Social y Totalidad

imposibilidad de la investigación social de abarcar la situación total o de atender a todos los hechos, entendiendo por todo o totalidad lo inverso a lo señalado anteriormente. Como ejemplos de ello podemos citar las posiciones de Mac Iver, de Johnson, de Parsons, y más recientemente de Boudón. Comencemos con la opinión del primero: "Nos damos cuenta que el establecimiento de las relaciones de causa a efecto llega a ser inmanejable si tenemos que atribuir a cada fenómeno, cada hecho, a la situación total de la cual emerge. Equivale a decir que el todo es la causa del todo, una afirmación tan inútil como su correlativo que el todo es el efecto del todo" (Mac Iver 1949:40).

Una visión semejante nos transmite Johnson: "No podemos ocuparnos simultáneamente de todos los cambios que se producen en un sistema social. Estamos obligados a considerar el impacto de cierto impulso al cambio - una perturbación del sistema, una tensión o conflicto, o un impulso cultural o ambiental de alguna especie- sobre una parte del sistema total, y al hacerlo consideramos temporariamente el resto del sistema como fijo o constante" (Johnson 1967:51).

Al reflexionar sobre el método de aproximación al estudio del cambio social, Parsons reconoce los mismos obstáculos: "Uno de los cánones fundamentales del método científico es que resulta imposible estudiarlo todo de una vez. Puesto que en la ciencia la base de la generalización es siempre la demostración de relaciones en el proceso de variación (de cambio en determinado sentido), debe haber siempre en alguna parte una distinción entre los rasgos de los fenómenos observados que cambian y los que no cambian dentro de las limitaciones pertinentes de tiempo y alcance y en los respectos considerados importantes para los propósitos que se tienen entre manos. Las especificidades del cambio importante ni siquiera podrían identificarse si no hubiera un fondo relativo inmutable con el cual relacionarlas" (Parsons 1964:84).

Por su parte, Boudon nos advierte de la inadecuación de la noción de totalidad para el análisis del cambio social, en la medida que involucra como condición de la teoría una visión de todos los hechos: “Aplicada al análisis del cambio, la noción de totalidad puede implicar además que este último no pueda ser comprendido más que por referencia al conjunto de elementos constitutivos de la realidad social" (Boudon 1969:29). Para éste último, apenas tiene sentido querer explicar el cambio mediante la totalidad de la realidad social, ya que se trataría de una noción límite desprovista de significación operativa (Boudon 1969:31). Al argumentar su posición, Boudon no duda en afirmar que incluso Marx, Weber y Comte (a quienes considera los principales teóricos del cambio al nivel de las sociedades globales) no tomaron en consideración todos los niveles de profundidad para explicar el cambio, sino que privilegiaron un cierto número de variables (Boudon 1969:30).

Desde una perspectiva de totalidad abierta y situacional, es evidente la necesidad de rechazar las diferentes visiones citadas arriba. Ahora bien, lo que en todos los casos habría que evitar son los falsos antagonismos. Si por totalidad no se entendiera todos los hechos sino el conjunto de los hechos referenciados en primera instancia desde el actor social y fijados a las condiciones de identificación y análisis de una situación social concreta y limitada, podríamos validar en buena medida dichas afirmaciones, dado que efectivamente hay obstáculos insuperables para captar la complejidad de los procesos sociales, así como hay necesidad de una estrategia de priorización de los elementos que se observan y analizan.

Creemos que el desacuerdo conceptual no desmerece atender a la idea que postula Mac Iver que la explicación causal no necesita comprender todo el amplio mundo, sino más bien cómo la diferencia en el consiguiente depende de la diferencia en el antecedente (Mac Iver 1949:60). La mirada que proponemos igualmente nos aproxima a la posición de Adorno, cuando nos advierte sobre el carácter situado y en cierta 
forma apropiable de la realidad social, al afirmar que la lógica de constitución de la totalidad social, asociada a la naturaleza social del conflicto que se regenera en primera instancia en el momento individual (Adorno 2004:511).

En resumidas cuentas, a diferencia de lo que señala Boudon, consideramos que una teoría del cambio social permite aprehender la totalidad social, en tanto comprendamos a esta última como perspectiva y no como "todos los hechos sociales". Decimos por lo tanto que la teoría del cambio social puede involucrar la totalidad social como perspectiva. La totalidad no implica todos los hechos sino sólo aquellos que consideramos que define una determinada realidad concreta y situada. En este sentido, podríamos entender la totalidad como totalidad situacional. Tal y como la concebimos, la totalidad es un proceso abierto y por ello nunca logrado plenamente, que nos devuelve de forma permanente a la historicidad de las totalizaciones que la determinan.

\section{(In)conclusiones}

La práctica desaparición pública de los estudios sobre el cambio social es en todo sentido un acontecimiento histórico, fundado sobre una crítica radical a las teorías de la modernidad. La recuperación de la pregunta por el cambio social desde la teoría social remite a la necesidad de revisión de la perspectiva marxiana, y de los diferentes enfoques marxistas y neomarxistas, así como de las de las corrientes funcionalistas, dominantes en el mundo occidental durante la segunda mitad del siglo 20.

Es necesario destacar la importancia epistemológica del concepto de totalidad como categoría central del método de investigación del cambio social. Aproximarse a cierta explicación del cambio social implica la superación de las visiones parceladas y fragmentadas del proceso social, a partir de una mirada reflexiva sobre la realidad como un todo. Es necesaria la apropiación de un esquema que permita hacer referencia al conjunto de los elementos que arbitrariamente reconocemos como constituyentes de lo social y de su transformación en una situación concreta.

Junto a la posibilidad de superar la visión compartimentada de los procesos sociales, una visión de totalidad (en los términos dialécticos de Adorno) permitiría incluso razonar esa división como producto de una forma histórica específica de regulación social de los objetos, hechos y relaciones sociales.

El hecho de asumir la teoría del cambio social como una perspectiva de totalidad situada o situacional (en los términos de una sociología del conocimiento) invalida la distinción entre teorías parciales y teorías totales del cambio social, elaborada como material polémico por representantes de la corriente del funcionalismo estructuralista, especialmente en su diálogo con el marxismo. Ahora bien, si aceptamos la posibilidad de concebir una relación jerárquica y de confrontación entre la idea de sociedad y la de cambio social, podemos dar cabida al prejuicio funcionalista, y concebir eventualmente el carácter subalterno de la teoría del cambio social respecto a la teoría social general.

Finalmente, cuando decimos que todo esfuerzo sociológico tiene que contentarse con razonar sobre una zona limitada del cambio social, debemos añadir que las limitaciones de cada aproximación responden a la represión que ejerce el propio concepto del cambio social que suscribimos y que simultáneamente consideramos inabarcable, junto a los constreñimientos de la propia realidad estructural del espacio social. Igualmente, el conocimiento del cambio social no puede erradicar su composición ideológica (si es que hubiese voluntad para hacerlo), sino sólo combatirla para intentar reducirla a su mínima expresión. 
A partir de constatar que el problema del cambio social (y la totalidad) se define en diálogo con la filosofía de la historia, presuponemos y luego asumimos una relación estrecha de trabajo entre ciencias sociales y filosofía. Ello implica, en los términos aquí expuestos, dejar de lado el formalismo ahistórico, y asumir los problemas sociales desde una interpretación global del proceso histórico. Nuestra intención metodológica se orienta por ello a "contaminar" los estudios sociológicos con materiales histórico-filosóficos. A partir de asumir que todo lo social es histórico y todo lo histórico es social, también buscamos reducir las diferencias analíticas y convencionales muchas veces difusas entre los estudios del cambio social y las investigaciones sobre cambio histórico.

\section{Notas}

(1) El artículo se desarrolla en el marco del proyecto de investigación "Lo imaginario como hipótesis sociológica. Naturaleza, matices e implicancias de la noción de imaginario social respecto de dos problemas sustantivos de la teoría sociológica", dirigido por el Dr. Javier Cristiano (CIECS-CONICET), financiado por SECYT-Universidad Nacional de Córdoba (Argentina), período 2009-2011.

(2) Este trabajo se articula con un texto anterior de mi autoría, titulado "Cambio social y determinación", publicado en la revista Acta Sociológica, UNAM, México, Mayo-Agosto de 2010, pp.45-75.

(3) Si bien supera los objetivos y las pretensiones del presente trabajo, es menester indicar que la iniciación de un diálogo sistemático e histórico con las principales doctrinas contemporáneas de pensamiento del cambio social en América Latina, como son, por ej., las teorías de la dependencia, la teología de la liberación -particularmente del trabajo de Enrique Dussel-, la pedagogía de Paulo Freire, la psicología social de Pichon Riviere, etc., es un paso necesario para poder definir un pensamiento situado desde la totalidad y referido al cambio social.

(4) Para profundizar en la relación entre totalidad y dialéctica, consultar Riu 1968.

(5) Sobre los alcances y las implicancias de la totalidad dialéctica de Adorno, consultar Caggiano 2007.

\section{Bibliografía}

Adorno, T.W. 2004. Escritos sociológicos I. Madrid: Akal.

Appelbaum, R. y Chambliss, W. 1997. Sociology: A brief introduction. New York: Longman.

Badiou, A. 2006. La potencia de lo abierto: universalismo, diferencia e igualdad. Archipiélago 73/74: 21-34.

Benjamin, W. 2004. El libro de los pasajes. Madrid: Akal.

Boudon, R. 1969. Los métodos en sociología. Barcelona: Aredondo.

Boudon, R. 1985. La place du desordre. Critique des theories du changement. Paris: Presses Universitaries de France.

Caggiano, S. 2007. Lecturas desviadas sobre comunicación y cultura. La Plata, Argentina: UNLP.

Ciurana, E. R. 1999. O pensar complexo. Edgar Morin e a crise da modernidade. Río de Janeiro: Garamond. 
Dussel, E. 1974. Método para una filosofía de la liberación. Salamanca: Sígueme.

Eisenstadt, S.N. 1970. Ensayos sobre el cambio social y la modernización. Madrid: Tecnos.

Etzioni, A. 1964. Epigénesis de la unificación política. En Etzioni, A. Los cambios sociales. México: Siglo XXI, México, pag. 428-442.

Francisco, A. 1997. Sociología y cambio social. Barcelona: Ariel.

Giddens, A. 1984. La constitución de la sociedad. Bases para una teoría de la estructuración. Buenos Aires: Amorrortu.

Grunner, E. 2002. El fin de las pequeñas historias: De los estudios culturales al retorno (imposible) de lo trágico. Buenos Aires: Paidós.

Harrison, D. 1988. The sociology of modernization and development. New York: Taylor.

Jaguaribe, H. 1972. Sociedad, cambio y sistema político. Buenos Aires: Paidós.

Johnson, H. M. 1967. El cambio social. Buenos Aires: Paidós.

Kosik, K. 1963. Dialéctica de lo concreto. México: Grijalbo.

Kuhn, T. 2005. La estructura de las revoluciones científicas. Madrid: Fondo de Cultura Económica.

Levinas, E. 2002. Totalidad e infinito. Ensayo sobre la exterioridad. Salamanca: Sígueme.

London, S. 1996. Understanding change: How it happens and how to make it happen. Fuente: http://www.scottlondon.com/reports/change.html. Consultado el 15 de septiembre de 2011.

Mac Iver, R. 1949. La causación social. México: Fondo de Cultura Económica.

Mc Michael, P. 2004. Development and social change: A global perspective. London and New Delhi: Sage Publications.

Moore, W. 1966. Cambio social. México: Uteha.

Morrow, R. 1995. Theories of social change. Canada: University of Alberta Press.

Nisbet, R. 1979. El problema del cambio social. En: Nisbet, R; Kuhn, T y White, L. Cambio social. Madrid: Alianza, pp. 12-51.

Parsons, T. 1964. Una teoría funcional del cambio. En: Etzioni, A. Los cambios sociales. México: Siglo XXI, pag. 84-96.

Pettigrew, T. y Back, K. 1967. La sociología en el proceso de desagregación. En Lazarsfeld, P; Sewell, W. y Wilensky, H. (comp.). La sociología y el cambio social. Buenos Aires: Paidós, pp. 62-96.

Puigbo, R. 1966. Cambio y desorganización social. Buenos Aires: Pleamar. 
Riu, F. 1968. Historia y totalidad. El concepto de reificación en Lukacs. Venezuela: Monteavila.

Roberts, T. y Hite, A. (eds.) 2000. Perspectives on development and social change. Oxford: Blackwell.

Sarlo, B. 2009. Verdad de los detalles. Trabajo y sociedad 12(11): 1-3.

Sartre, J. P. 1960. Crítica de la razón dialéctica. Buenos Aires: Losada.

Smelser, N. y Haferkamp, H. (eds.) 1992. Social change and modernity. Berkeley: University of California Press.

Sztompka, P. 1995. Sociología del cambio social. Madrid: Alianza.

Wallerstein, I. 1994. Agonías del capitalismo. Iniciativa socialista $31 . \quad$ Fuente: http://www.inisoc.org/Waller.htm. Consultado el 17 de Octubre de 2011.

Wright Mills, Ch. 1964. Las fuentes del poder de la sociedad. En: Etzioni, A. Los cambios sociales. México: Siglo XXI, pp. 119-125.

Y So, A. 1990. Social change and development; modernization, dependency and world system theory. London: Sage.

Recibido el 17 Jun 2011

Aceptado el 20 Oct 2011 